\title{
One-Dimensional Phenomenological Model for Liquid Water Flooding in Cathode Gas Channel of a Polymer Electrolyte Fuel Cell
}

Chaozhong Qin, S. Majid Hassanizadeh, Dirk Rensink and Stephan Fell

J. Electrochem. Soc. 2012, Volume 159, Issue 6, Pages B737-B745. doi: 10.1149/2.092206jes

\begin{tabular}{cc}
$\begin{array}{c}\text { Email alerting } \\
\text { service }\end{array}$ & $\begin{array}{l}\text { Receive free email alerts when new articles cite this article - sign up } \\
\text { in the box at the top right corner of the article or click here }\end{array}$ \\
\hline
\end{tabular}

To subscribe to Journal of The Electrochemical Society go to: http://jes.ecsdl.org/subscriptions 


\title{
One-Dimensional Phenomenological Model for Liquid Water Flooding in Cathode Gas Channel of a Polymer Electrolyte Fuel Cell
}

\author{
Chaozhong Qin, ${ }^{\text {a,b }}$ S. Majid Hassanizadeh, ${ }^{\mathrm{b}}$ Dirk Rensink, ${ }^{\text {a, }}$ and Stephan Fell ${ }^{\mathrm{a}}$ \\ ${ }^{a}$ Adam Opel AG, GME Engineering, GM Alternative Propulsion Europe, D-65432 Rüsselsheim, Germany \\ ${ }^{b}$ Department of Earth Sciences, Utrecht University, 3508 Utrecht, The Netherlands
}

\begin{abstract}
The mathematical description of liquid water flooding in the gas channel (GC) of a polymer electrolyte fuel cell (PEFC) at the macro scale has remained a challenge up to now. The mist flow assumption in the GC has been commonly used in previous numerical studies. In this work, a one-dimensional (down-the-channel) macroscale phenomenological model for the liquid water flooding in the cathode GC is developed based on several reasonable assumptions. We focus on the operating conditions with fully humidified inlet air on the cathode side. Some simplifications are introduced to obtain a manageable numerical model. A series of case studies are conducted to investigate the effects of droplets and various operating parameters on the liquid water flooding in the GC. The results show that the gas drag force at the film-gas interface significantly enhances the film flow along the GC corners. Based on a proposed droplet model, we can capture key scenarios of liquid water flooding in the GC under different operating conditions like varying stoichiometry, current density and cell temperature. We find that droplets in the GC mainly contribute to the gas pressure loss and water flooding in the GC, which should be depressed for better water management. At last, a proper selection of the GC sidewall contact angle is found to be critical to a better water management in the GC.

(C) 2012 The Electrochemical Society. [DOI: 10.1149/2.092206jes] All rights reserved.
\end{abstract}

Manuscript submitted December 6, 2011; revised manuscript received March 21, 2012. Published April 10, 2012.

Water management plays a critical role in the development of polymer electrolyte fuel cells (PEFCs). The proton exchange membrane needs to attain high water content for effective ionic conduction. On the other hand, too much liquid water accumulating inside porous layers results in reactant starvations and cell degradations. Such a situation is referred to as "flooding" in PEFCs. In order to balance both water requirements, a deep understanding of liquid water transport in a whole PEFC is needed. ${ }^{1-5}$

The cathode side of a PEFC is prone to being flooded first, since water is generated in the cathode catalyst layer (CL) due to electrochemical reactions. Then, excessive liquid water penetrates through the porous layers into gas channels (GCs). Typically, a GC is confined by three walls from the bipolar plate which are hydrophilic, and the surface of the gas diffusion layer (GDL) which is hydrophobic. When liquid water breaks through the GDL-GC interface, water droplets and film slugs form on the GDL surface and along hydrophilic GC corners, ${ }^{6-9}$ respectively. It is obvious that under flooding situations, liquid water transport on the cathode side can occur in both porous layers and micro GCs simultaneously.

Experimentally, several techniques have been employed to visualize liquid water evolution and transport inside porous layers, such as synchrotron $\mathrm{X}$-ray, ${ }^{10-12}$ neutron radiography ${ }^{13-16}$ and tomography, magnetic resonance imaging, ${ }^{17}$ as well as fluorescence microscope. ${ }^{18,19}$ As a result, a new liquid water transport mechanism through fibrous GDL is proposed, which is termed as "fingering and channeling" flow. ${ }^{18,20}$ Liquid water dynamics in the GC is quite complex due to its microstructure and mixed-wettability. Over the past few years, optical photography using transparent fuel cells ${ }^{7,8,21,36}$ have been widely used to study liquid water flow in the GC and droplet dynamics. As for computational fluid dynamics (CFD) simulations of liquid water transport in a PEFC, two major challenges lie in how to effectively mimic liquid water transport in the GC, and how to couple liquid water transport between the GDL and GC. Even though some interface tracking methods ${ }^{22-24}$ have been extensively used to investigate water dynamics in the GC, they all suffer from heavy computational efforts, and cannot be applied to a large level. In addition, these methods are based on a much smaller spatial scale than that in the GDL, where the averaged two-phase Darcy's law ${ }^{25}$ is commonly employed to describe the liquid water transfer. To date, there are only a few works addressing the interfacial coupling effect ${ }^{26-28}$ on the

${ }^{\mathrm{z}}$ E-mail: dirk.rensink@de.opel.com liquid water transport in porous layers, often based on some simplified models.

In this work, we mainly contribute to the first challenge mentioned above, and try to describe the liquid water transport in the GC at a macroscopic scale. We develop a model that is very computationally effective. Fig. 1 shows the schematic water flooding in the GC. With continuous liquid water filling from the GDL, film flow forms along the GC corners, and droplets sit on the GDL surface. Note that the isolated GC is our study domain to avoid the GDL-GC interface treatment as a first attempt.

In what follows, we first list the main assumptions used in the model development. Then, the detailed mathematical derivations are given. Finally, several case studies are conducted to investigate the effects of various engineering and operating parameters on the liquid water flooding in the GC, and some useful conclusions are obtained.

\section{Physical Model}

In this section, we first give the main assumptions adopted in this work. Then the governing equations of the phenomenological model for the water flooding in the GC are derived in detail.

Assumptions. - To make mathematical descriptions of water flooding in the GC feasible, and based on physical considerations, the following main assumptions are used in the model development:

1. The dynamic evolution of individual droplet in the GC can be described by the following sequential processes: emergence, growth, detachment, coalescence and finally wicking into the hydrophilic sidewalls of the GC (as shown in Fig. 2). In other words, droplets in the GC are not tracked explicitly.

2. The liquid water coming from the GDL, and condensed from water vapor flows out of the GC only via the film forming along the GC corners. This is a reasonable assumption under low gas flow rates..$^{8,9,29}$

Governing equations.- Fig. 3 depicts the assumed idealized liquid water distribution in the GC cross section, which is separated into film (including two symmetric parts) forming at the hydrophilic GC corners, as well as droplet sitting on the hydrophobic GDL surface (i.e. GC down wall in this work). Since droplet dynamics is not considered here, we focus on the derivations of governing 


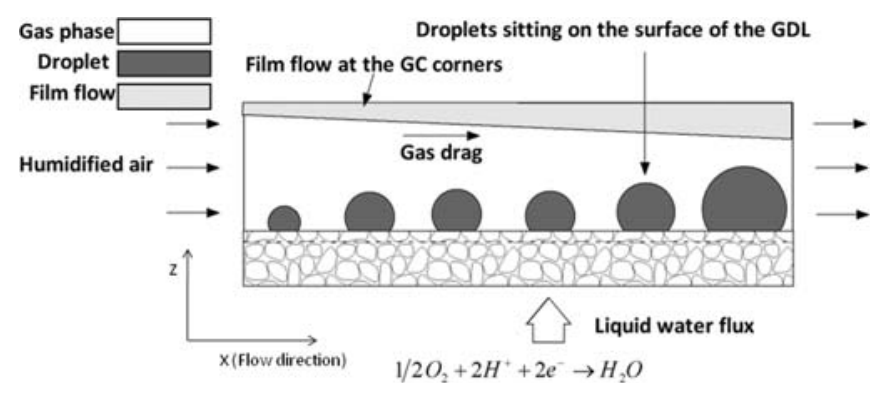

Figure 1. Two-dimensional schematic of liquid water flooding in the GC.

equations for the gas and film flows in what follows. The droplet effect in the GC is accounted for in terms of one parameter called droplet area in the GC cross section, $A_{d}$, which could be a function of $\mathrm{x}$ coordinate (flow direction along the GC) and other flow variables.

Gas phase.-We integrate the microscopic continuity equation for the gas phase over the GC cross section shown in Fig. 3, and get:

$$
\frac{d\left(A_{g} \rho_{g} v_{g}\right)}{d x}=S_{g}
$$

where $A_{g}$ is the area occupied by the gas phase in the GC cross section, $\rho_{g}$ is the gas mass density, $v_{g}$ denotes the area-averaged gas velocity, and $S_{g}$ represents the source/sink term of the gas phase owing to oxygen consumption and phase change between water vapor and liquid water. The gas flow direction is in the positive $\mathrm{x}$.

On the cathode side of a PEFC, humidified air is delivered into the GC. We have the following averaged species transport equations for both water vapor and oxygen:

$$
\begin{gathered}
\frac{d\left(A_{g} \rho_{g} C_{O_{2}} v_{g}\right)}{d x}=\frac{d}{d x}\left(A_{g} \rho_{g} D_{O_{2}} \frac{d C_{O_{2}}}{d x}\right)+S_{O_{2}} \\
\frac{d\left(A_{g} \rho_{g} C_{\mathrm{H}_{2} O} v_{g}\right)}{d x}=\frac{d}{d x}\left(A_{g} \rho_{g} D_{\mathrm{H}_{2} O} \frac{d C_{\mathrm{H}_{2} O}}{d x}\right)+S_{\mathrm{H}_{2} O}
\end{gathered}
$$

Here, $C_{\mathrm{O}_{2}}$ and $C_{\mathrm{H}_{2} \mathrm{O}}$ denote the mass fractions of oxygen and water vapor, respectively, $D_{\mathrm{O}_{2}}$ and $D_{\mathrm{H}_{2} \mathrm{O}}$ are the diffusivities of oxygen and water vapor, respectively, $\mathrm{S}_{\mathrm{O}_{2}}$ is the oxygen sink term, and $\mathrm{S}_{\mathrm{H}_{2} \mathrm{O}}$ is the water vapor sink/source term.

The relation between the gas velocity and gas pressure gradient along the GC is approximated by the well-known Hagen-Poiseuille equation, ${ }^{30}$ which is given as:

$$
v_{g}=-K_{g}\left(\mu_{g}, A_{g}\right) \frac{d p_{g}}{d x}
$$

where $p_{g}$ is the gas pressure, and $K_{g}$ is called the flow conductivity of the gas phase, which is a function of gas dynamic viscosity and gas flow area. With the help of the hydraulic diameter for the gas flow, the

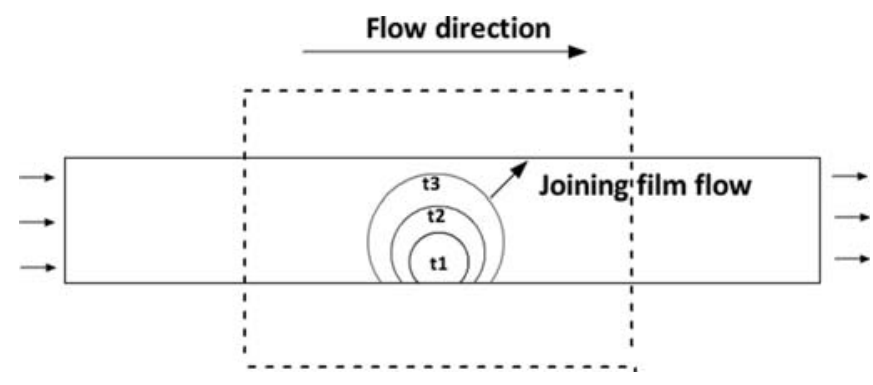

Figure 2. Schematic of the growth of a water droplet with time in the GC (Finally, this droplet reaches to a critical size comparable to the GC dimensions, and joins the film flow along the GC corners).

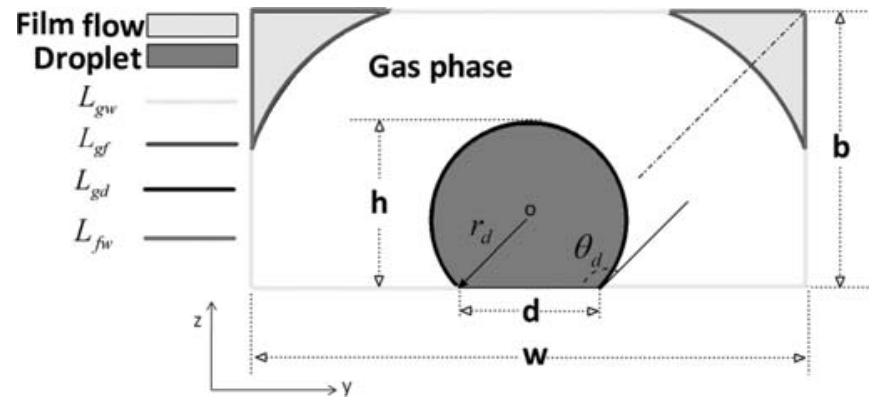

Figure 3. Assumed liquid water distribution at the GC cross section, including the film at the corners and the water droplet sitting at the center.

gas flow conductivity is specified as follows:

$$
\begin{gathered}
K_{g}=\frac{D_{H}^{2}}{32 \mu_{g}}=\frac{1}{32 \mu_{g}}\left(\frac{4 A_{g}}{L_{n o-s l i p}}\right)^{2} \\
L_{n o-s l i p}=L_{g w}+L_{g f}+L_{g d}
\end{gathered}
$$

In Eq. $5, D_{H}=4 A_{g} / L_{n o-s l i p}$ is the hydraulic diameter for the gas flow, and $L_{n o-s l i p}$ is the wetted perimeter of the gas flow area as shown in Fig. 3, which is given in Eq. 6. $L_{g w}, L_{g f}$ and $L_{g d}$ denote the lengths of gas-wall interface, gas-film interface, as well as gas-droplet interface, respectively.

Isothermal condition is assumed in this work, no energy equation for gas or film phase is needed. In addition, we have the following three complementary equations for the gas phase:

$$
\begin{gathered}
A_{g}+A_{f}+A_{d}=A_{g c} \\
C_{O_{2}}+C_{H_{2} O}+C_{N_{2}}=1 \\
\rho_{g}=\frac{p_{g}}{R T \sum_{i} \frac{C_{i}}{M_{i}}}
\end{gathered}
$$

Eq. 7 means that the summation of the gas phase area, film area, and droplet area is equal to the GC cross-sectional area, which can be seen in Fig. 3. Eq. 8 shows the summation of species mass fractions equals to unity. Finally, Eq. 9 is the state equation for an ideal gas mixture, where $C_{i}$ is the species mass fraction, $M_{i}$ is the molecular weight of each species, $R$ is the universal gas constant, and $T$ denotes the cell operating temperature.

Film phase.-The averaged continuity equation for the film phase is given as:

$$
\frac{d\left(A_{f} \rho_{f} v_{f}\right)}{d x}=S_{f}
$$

where $v_{f}$ is the average velocity of the film phase, and $S_{f}$ denotes the source term due to water flux from the GDL as well as phase change between water vapor and film.

Based on the average process, the film flow equation can be expressed as follows:

$$
v_{f}=-\frac{r_{f}^{2}}{\mu_{f} \beta} \frac{d p_{f}}{d x}+F_{d r a g} \frac{r_{f} \tau}{\mu_{f}}
$$

where $r_{f}$ is the radius of the curvature of gas-film interface, $\mu_{f}$ is the dynamic viscosity of liquid water, $p_{f}$ denotes the film pressure, $\tau$ represents the gas shear stress at the gas-film interface, $\beta$ is called dimensionless viscous resistance, and $F_{d r a g}$ can be called dimensionless gas drag coefficient. Greater detail on the derivation of Eq. 11 is given in Appendix A. 


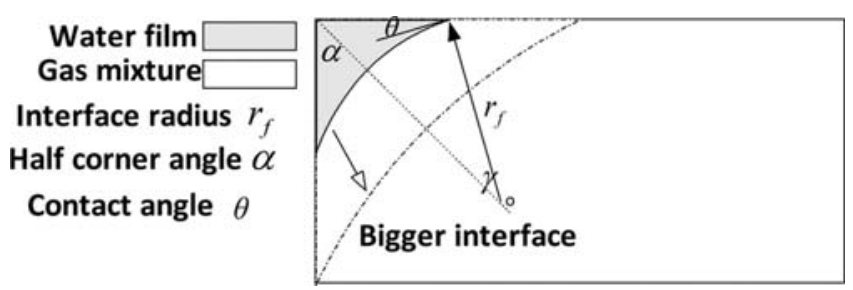

Figure 4. Schematic of the film configuration at a GC corner.

We can find the approximate expressions for $\beta$ and $F_{\text {drag }}$ by means of numerical simulations. Particularly, extensive studies on the dimensionless viscous resistance are available in literature. ${ }^{32}$ The two pressures for the gas and film phases are coupled through the capillary pressure:

$$
p_{c}=p_{g}-p_{f}=\frac{\sigma}{r_{f}}
$$

Here, $\sigma$ represents the surface tension between gas and liquid water, and we assume that the radius of the curvature of gas-film interface along the GC is infinite.

Geometrical information.-In order to close the governing equations derived above, some additional equations are required which can be obtained based on the geometrical information shown in Fig. 3 and Fig. 4. The Concus and Finn condition ${ }^{33}$ should be satisfied in this work to ensure a stable film distribution along the GC corners:

$$
\alpha+\theta<\pi / 2
$$

The film area in the GC cross section can be written as a function of the radius of the curvature of gas-film interface:

$$
A_{f}=r_{f}^{2}\left[\sin (2 \gamma)+\frac{2 \sin ^{2} \gamma}{\tan \alpha}-2 \gamma\right]
$$

where $\alpha$ is the upper half corner angle of the GC, and $\gamma$ is expressed as:

$$
\gamma=\frac{\pi}{2}-\alpha-\theta
$$

where $\theta$ denotes the static contact angle of the GC sidewalls.

The areas of droplet and GC cross sections are given as:

$$
\begin{gathered}
A_{d}=r_{d}^{2}\left[\theta_{d}-\frac{\sin \left(2 \theta_{d}\right)}{2}\right] \\
A_{g c}=b \sin (\pi-2 \alpha)[w-b \cos (\pi-2 \alpha)]
\end{gathered}
$$

in which, $\theta_{d}$ denotes the static contact angle of the GDL surface, and $r_{d}$ is the droplet radius shown in Fig. 3. In Eq. 17, $b$ is the side edge length of the GC cross section, and $w$ is the down edge length of the GC cross section.

As shown in Fig. 3, the lengths of the gas-film interface, gas-wall interface, gas-droplet interface, and film-wall interface can be given as:

$$
\begin{gathered}
L_{g f}=4 r_{f} \gamma \\
L_{g d}=2 r_{d} \theta_{d}
\end{gathered}
$$

$$
L_{g w}=2 w+2 b[1-\cos (\pi-2 \alpha)]-2 r_{d} \sin \left(\pi-\theta_{d}\right)-\frac{4 r_{f} \sin \gamma}{\sin \alpha}
$$

$$
L_{f w}=\frac{4 r_{f} \sin \gamma}{\sin \alpha}
$$

\section{Numerical Model}

Simplifications of governing equations. - As a first attempt, we make some simplifications of the governing equations derived above for numerical studies. First, we focus on the fully humidified air inlet on the cathode side. Therefore, we just need to consider the water vapor condensation along the GC. Constant molar density of the gas phase is assumed in the GC due to the relatively small pressure drop, which is given as:

$$
\widehat{\rho}_{g}=\frac{p_{0}}{R T}
$$

where $\hat{\rho}_{g}$ designated by the caret is the molar density of the gas phase, and $p_{0}$ is the gas inlet pressure.

We also neglect the species diffusion in the GC and simplify the transport equations 2 and 3 as follows:

$$
\begin{gathered}
\frac{d\left(A_{g} \widehat{\rho}_{\mathrm{O}_{2}} v_{g}\right)}{d x}=\widehat{S}_{\mathrm{O}_{2}} \\
\frac{d\left(A_{g} \widehat{\rho}_{\mathrm{H}_{2} \mathrm{O}} v_{g}\right)}{d x}=\widehat{S}_{\mathrm{H}_{2} \mathrm{O}}
\end{gathered}
$$

Here, $\widehat{\rho}_{\mathrm{O}_{2}}$ and $\widehat{\rho}_{\mathrm{H}_{2} \mathrm{O}}$ represent the molar densities of oxygen and water vapor, respectively, and $\widehat{S}_{\mathrm{O}_{2}}$ and $\widehat{S}_{\mathrm{H}_{2} \mathrm{O}}$ are the molar source/sink terms $(\mathrm{mol} / \mathrm{m} / \mathrm{s})$.

Since the inlet gas is fully humidified, we can write the molar density of water vapor as:

$$
\widehat{\rho}_{H_{2} O}=\frac{p_{\text {sat }}}{R T}
$$

where $p_{\text {sat }}$ is the saturated water vapor pressure, given by the following empirical expression ${ }^{34}$ :

$$
\begin{aligned}
\log _{10}^{p_{\text {sat }} / 101325}= & -2.1794+0.02953(T-273.15)-9.1837 \\
& \times 10^{-5}(T-273.15)^{2}+1.4454 \\
& \times 10^{-7}(T-273.15)^{3}
\end{aligned}
$$

After several algebraic manipulations on Eq. 22-25, the molar sink term of water vapor is expressed as:

$$
\widehat{S}_{\mathrm{H}_{2} O}=\frac{P_{\text {sat }}}{P_{0}-P_{\text {sat }}} \widehat{S}_{\mathrm{O}_{2}}
$$

So, the gas phase averaged continuity equation can be obtained as:

$$
\frac{d\left(A_{g} v_{g}\right)}{d x}=\left(\frac{R T}{p_{0}-p_{s a t}}\right) \hat{S}_{O_{2}}
$$

The molar sink term of oxygen is given as:

$$
\widehat{S}_{O_{2}}=-\frac{I(x) w}{4 F t}
$$

where $I(x)$ is the cell current density as a function of $\mathrm{x}$ coordinate, $F$ is the Faraday constant, $w$ is the down edge length of the GC cross section, and $t$ denotes the ratio of the area of the GC down wall to the reactive area in the membrane.

For the film phase, the continuity equation 10 may be rewritten as:

$$
\frac{d\left(A_{f} v_{f}\right)}{d x}=\frac{S_{f}}{\rho_{f}}=\frac{M_{H_{2} O}}{\rho_{f}}\left[\frac{(1+2 a) I(x) w}{2 F t}-\widehat{S}_{H_{2} O}\right]
$$

In Eq. 30, the film density is assumed to be constant, $M_{H_{2} O}$ denotes the molecular weight of water vapor, and $a$ is the net water transfer coefficient.

Substituting Eq. 12 into Eq. 11, we get the film flow equation as:

$$
v_{f}=-\frac{r_{f}^{2}}{\mu_{f} \beta} \frac{d p_{g}}{d x}-\frac{\sigma}{\mu_{f} \beta} \frac{d r_{f}}{d x}+F_{d r a g} \frac{r_{f} \tau}{\mu_{f}}
$$


A simplified expression for the dimensionless viscous resistance $\beta$, based on an analytical solution, is available in the literature, ${ }^{35}$ which is given as:

$$
\begin{gathered}
\beta=\frac{12 \sin ^{2} \alpha(1-B)^{2}}{(1-\sin \alpha)^{2} B^{2}} \frac{\left(\psi_{1}-B \psi_{2}\right) \psi_{3}^{2}}{\left(\psi_{1}-B \psi_{2}\right)^{3}} \\
B=\left(\frac{\pi}{2}-\alpha\right) \tan \alpha \\
\psi_{1}=\cos ^{2}(\alpha+\theta)+\cos (\alpha+\theta) \sin (\alpha+\theta) \tan \alpha \\
\psi_{2}=1-\frac{\theta}{\frac{\pi}{2}-\alpha} \\
\psi_{3}=\frac{\cos (\alpha+\theta)}{\cos \alpha}
\end{gathered}
$$

The gas shear stress at the gas-film interface can be formulated as:

$$
\tau=-f_{\tau} \frac{A_{g}}{L_{n o-s l i p}} \frac{d p_{g}}{d x}
$$

In Eq. 37, $f_{\tau}$ denotes the effect of the gas flow configuration on the shear stress distribution, which can be obtained based on numerical experiments $^{31}$ :

$$
f_{\tau}=1-\exp \left[\frac{-13 r_{f}}{D_{H}}\left(\cos \gamma+\frac{\sin \gamma}{\tan \alpha}-1\right)\right]
$$

We also can employ numerical simulations to obtain values for the dimensionless gas drag coefficient, which is given in Table II. Note that the effect of the contact angle of the GC sidewalls is neglected here.

Boundary conditions and numerical implementation.- Based on the adopted simplifications in Section 3.1, five first-order ordinary differential equations (ODEs) are obtained, namely, Eq. 28, Eq. 23, Eq. 4, Eq. 30, and Eq. 31, associated with five unknown variables, $v_{g}$, $\hat{\rho}_{\mathrm{O}_{2}}, p_{g}, v_{f}$, and $r_{f}$. In the following, we describe the applied boundary conditions.

The inlet gas velocity is decided by averaged current density, stoichiometric ratio, reactive area, as well as GC cross-sectional area, expressed as:

$$
\left.v_{g}\right|_{x=0}=\frac{\xi_{c} I_{a} A_{m}}{4 F \widehat{\rho}_{O_{2}} A_{g}}=\frac{\xi_{c} A_{m}}{4 L F\left(\left.\hat{\rho}_{O_{2}}\right|_{x=0}\right) A_{g}} \int_{0}^{L} I(x) d x
$$

where $\xi_{c}$ is the stoichiometric ratio on the cathode side, $L$ is the GC length, $I_{a}$ is the averaged current density along the GC, and $A_{m}$ denotes the reactive area.

The inlet oxygen molar density is dependent on the inlet gas pressure and relative humidity:

$$
\left.\widehat{\rho}_{O_{2}}\right|_{x=0}=\frac{0.21 p_{0}}{R T}\left(1-\frac{p_{\text {sat }}}{p_{0}} R H\right)
$$

where $R H$ represents the relative humidity of the inlet air, and it is $100 \%$ in this work.

A constant inlet gas pressure is specified as:

$$
\left.p_{g}\right|_{x=0}=p_{0}
$$

The film velocity at the GC inlet is set to be zero:

$$
\left.v_{f}\right|_{x=0}=0
$$

\begin{tabular}{ll}
$\begin{array}{l}\text { Table I. Geometrical, physical and operating parameters for base } \\
\text { case. }\end{array}$ & Value \\
Parameter & $1.0 / 0.5 / 200 \mathrm{~mm}$ \\
\hline GC width/height/length & None \\
Droplet radius at the GC cross section, $r_{d}$ & $7 \pi / 9$ radian \\
Contact angle of the bottom wall of the & \\
GC, $\theta_{d}$ & 0 radian \\
Contact angle of the GC sidewalls, $\theta$ & $\pi / 4$ radian \\
Half angle of the GC corner, $\alpha$ & $6000 \mathrm{~A} \mathrm{~m}{ }^{-2}$ \\
Averaged current density, $I_{a}$ & 113.38 \\
Dimensionless viscous resistance, $\beta$ & 0.065 \\
Dimensionless gas drag coefficient, & \\
$F_{d r a g}$ & $353.15 \mathrm{~K}^{-}$ \\
Cell temperature, $T$ & $1.5 \mathrm{bar}^{-}$ \\
Inlet gas mixture pressure (or cell & \\
operating pressure), $p_{0}$ & $972 \mathrm{~kg} \mathrm{~m}^{-3}$ \\
Liquid water density, $\rho_{l}$ & $3.5 \times 10^{-4} / 2.03 \times 10^{-5} \mathrm{~Pa} \mathrm{~s}$ \\
Dynamic viscosity of liquid water/gas \\
mixture, $\mu$
\end{tabular}

For the radius of the curvature of the gas-film interface, we assume a fully developed boundary condition at the GC out, expressed as:

$$
\left.\frac{d r_{f}}{d x}\right|_{x=L}=0
$$

Since the five ODEs are highly nonlinear and coupled, MATLAB 2010 is employed to solve them numerically. Note that, good initial guesses of unknown variables are essential to solve the ODEs with boundary conditions in this work.

\section{Results and Discussion}

Table I lists the geometrical, physical and operating parameters for the base case. Note that we assume that droplets are absent in the base case. Fig. 5 shows the effects of the half corner angle of the $\mathrm{GC}$ and the contact angle of the GC sidewalls on the dimensionless viscous resistance, $\beta$. Generally speaking, the dimensionless viscous resistance increases with the increase of the GC half corner angle. For a given half corner angle, no obvious change of the dimensionless viscous resistance can be found with the change in the contact angle of the GC sidewalls, as long as the contact angle is smaller than 15 degrees. While the contact angle is larger than 15 degrees, the dimensionless viscous resistance increases dramatically as increasing the contact angle.

Base case study. - In the base case study, we suppress the appearance of droplets in the GC. This may be done by regulating liquid water flow into the GC. For more detail, one can refer to the reference. ${ }^{29} \mathrm{We}$ note that low water saturation in GCs is beneficial to the performance

Table II. Dimensionless gas drag coefficients for different GC upper half corner angles (adapted from the reference 31).

Half corner angle, $\alpha$ (radian) Dimensionless gas drag coefficient, $F_{d r a g}$

$\begin{array}{ll}\pi / 6 & 0.086 \\ 7 \pi / 36 & 0.08 \\ 2 \pi / 9 & 0.073 \\ \pi / 4 & 0.065 \\ 5 \pi / 18 & 0.055 \\ 11 \pi / 36 & 0.045 \\ \pi / 3 & 0.035\end{array}$




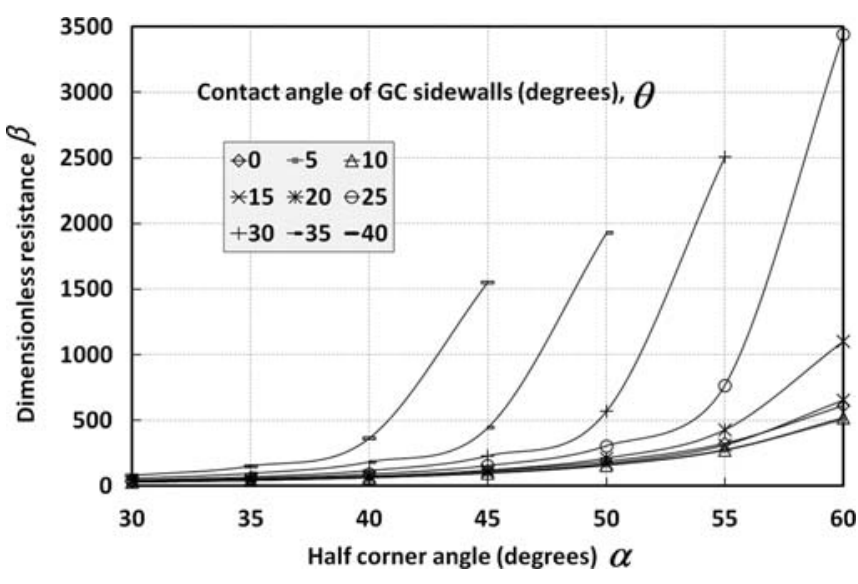

Figure 5. Effects of the half corner angle of the GC and the contact angle of the GC sidewalls on the dimensionless resistance.

of PEFCs due to low water coverage at the GDL-GC interface and small pressure losses along GCs. Fig. 6 shows the distribution of liquid water saturation along the flow direction. As a uniform water flux is provided through the bottom of the GC, more and more liquid water accumulates along the GC corners. As a result, the distribution of the film thickness along the GC is from thin to thick. Since no droplet is considered here, the liquid water saturation is quite small, only up to 0.031 at the end of the GC (see the insertion in Fig. 6).

The gas phase velocity decreases along the flow direction as shown in Fig. 7 (left). This can be explained by the fact that while the change of the gas flow area is quite small, the gas phase loses considerable mass along the GC due to the water vapor condensation and oxygen consumption. Fig. 7 (right) shows that the film velocity increases along the GC from 0 to about $0.024 \mathrm{~m} / \mathrm{s}$. This is due to the water vapor condensation and uniform liquid water flux from the GDL. It is seen that the film velocity is much smaller compared to that of the gas phase, which results in large gas drag force at the filmgas interface. This gas drag force assists the film flow along the GC corners considerably. ${ }^{29}$ Therefore, very low water saturation is found in the GC (see Fig. 6).

Fig. 8 (left) displays the oxygen molar density reduction along the GC. For the base case, the stoichiometric ratio is 2.0 , and the molar density of oxygen at the GC outlet is a little higher than half of that at the GC inlet mainly due to the decrease of gas phase velocity along the GC (Fig. 7). Fig. 8 (right) shows the gas pressure distribution along the GC relative to the inlet pressure (1.5 bar). The total pressure drop is about $911 \mathrm{~Pa}$, which is quite close to that for single-phase flow in

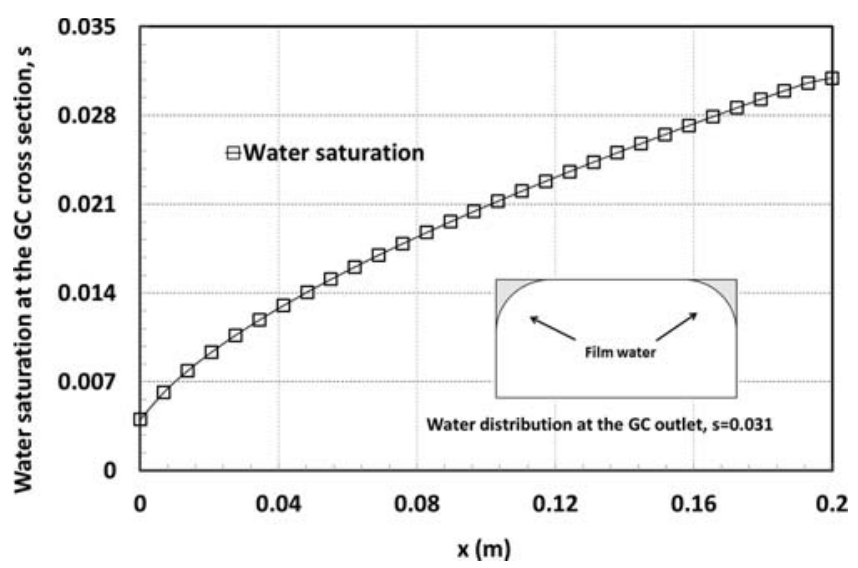

Figure 6. Distribution of liquid water saturation along the GC (Insertion: liquid water distribution at the GC outlet).

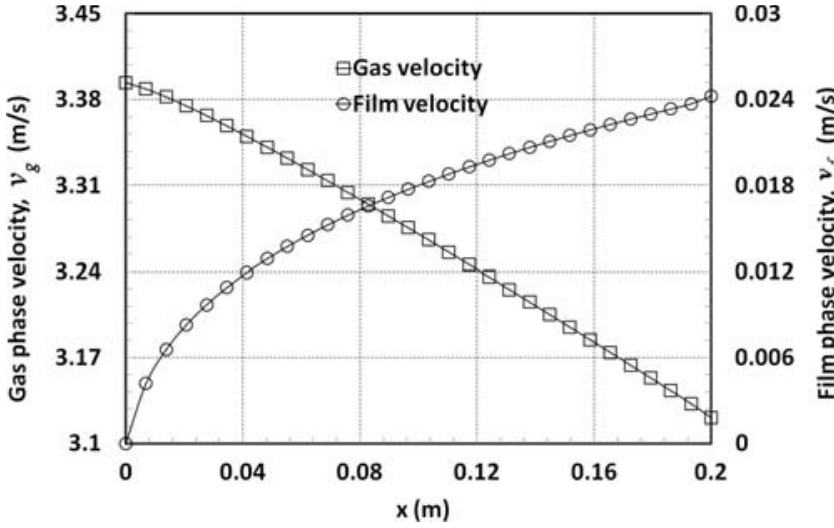

Figure 7. Left: gas phase velocity distribution along the GC (decreasing); right: film phase velocity distribution along the GC (increasing).

the GC. This indicates that film flow along the GC corners has little impact on gas pressure loss due to its small saturation.

Effect of droplets in the GC.- It has been assumed that droplets in the GC join in the film flow along the GC corners though a series of dynamic processes. However, at any time droplets can be always observed in the GC under flooding situations. For comprehensiveness, the effect of droplets should be taken into account in the present steadystate GC flooding model. Since droplets are not explicitly tracked, we introduce a parameter called droplet radius (see Eq. 16) in our model development. In addition, the droplet radius at the GC cross section (Fig. 3) can be expected to be a function of several variables such as Reynolds number of gas flow, current density, and contact angle of the GDL surface, as well as pore-structure of the GDL. We can illustrate it as follows:

$$
r_{d}=f\left(\operatorname{Re}, \theta_{d}, I, \text { etc }\right)
$$

Up to now, it is still difficult to derive a general explicit expression for droplet distribution along the GC. It should be possible, however, to obtain some empirical expressions by means of experimental observations. As a first attempt, we propose a simple empirical model for calculating the droplet radius at the GC cross section:

$$
r_{d}=k \frac{A_{g c}}{A_{g} v_{g}} I(x)
$$

In Eq. 45, we take into account two major factors influencing the magnitude of droplet radius: local water flux from the GDL denoted

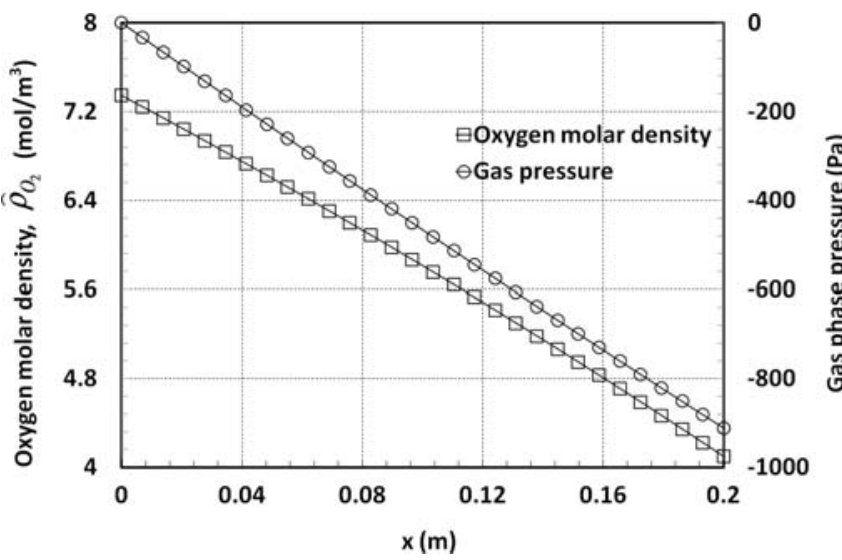

Figure 8. Left: oxygen molar density distribution along the GC; right: gas pressure drop along the GC. 


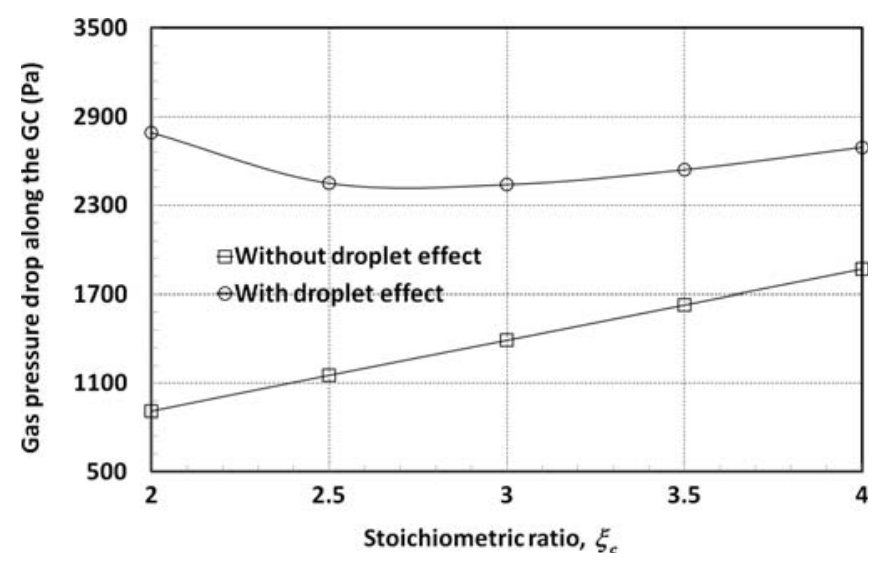

Figure 9. Droplet effect on gas pressure losses along the GC for different stoichiometric ratios.

by local current density $I(x)$ and local gas drag force approximated by gas flow rate. In addition, we note that the coefficient $k$ serves as a fitting parameter, which should be calibrated by experimental data. In principal, the coefficient $k$ should also be a dependent parameter. However, for simplicity in this work it is set to a constant of 1.0 $\times 10^{-7}$ due to the absence of experimental data.

Fig. 9 shows the droplet effect on gas pressure drops along the GC for different stoichiometric ratios. Note that expect for droplet radius and stoichiometry, all other parameters are the same as for the base case. It is seen that the gas pressure loss is almost linearly proportional to the applied stoichiometry when droplets are absent, which corresponds to the mist flow assumption used by most previous PEFC models. ${ }^{26,34}$ By incorporating the droplet model of Eq. 45, we obtain the totally different gas pressure losses along the GC as shown by the upper curve in Fig. 9. At the stoichiometry of 2.0, the gas pressure loss is up to about $2790 \mathrm{~Pa}$, which is about three times as large as that without droplet effect. As increasing the stoichiometry the gas pressure loss reduces first due to the enhanced gas drag force on the droplets. As a consequence, smaller droplets are expected in the GC. Later on the gas pressure loss increases slightly because of the enlarged gas flow rate, which starts to dominate the gas pressure loss along the GC. The same phenomenon was also observed in experiments ${ }^{39}$ which can be captured very well by the present GC flooding model.

Fig. 10 shows the water saturation and gas pressure distributions along the GC at the stoichiometry of 2.0. The water saturation at the GC cross section includes two contributions: film water and droplet

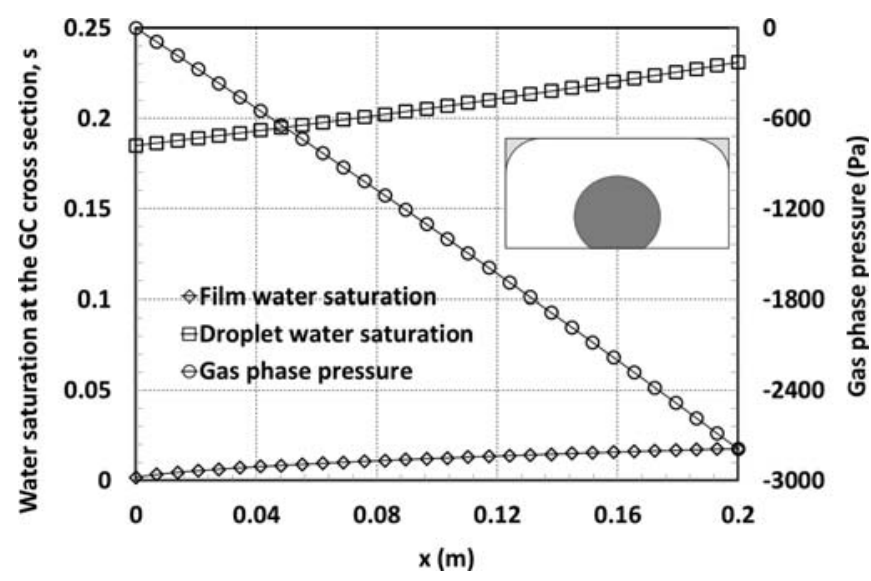

Figure 10. Distributions of water saturation and gas pressure along the GC at the stoichiometry of 2.0 (Insertion: liquid water distribution at the GC outlet, including both film and droplet).

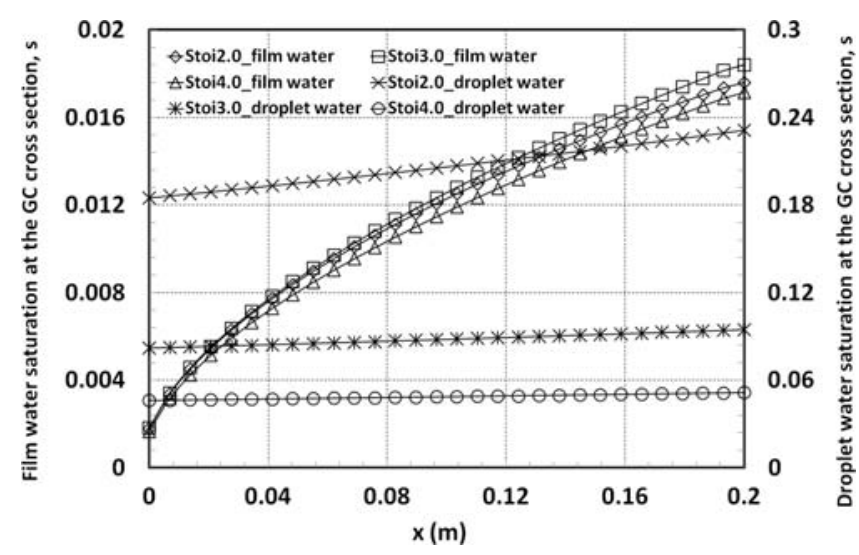

Figure 11. Distributions of water saturation (i.e. film water saturation and droplet water saturation) along the GC for different stoichiometric ratios.

water. It can be seen obviously that the droplet water saturation is much bigger than the film one, which increases slightly due to the decreased gas drag force. The gas pressure drops almost lineally along the GC mainly owing to the small variation of liquid water saturation in the GC. The insertion in Fig. 10 displays the liquid water distribution at the GC outlet with the film water saturation of about 0.02 and droplet water saturation of about 0.23 . It is evident that droplets in the $\mathrm{GC}$ are not only the main contributions to gas pressure loss, but also cover the GDL-GC interface blocking reactant diffusion into reactive sites. Therefore for better water management in GCs, we should depress the emergence of droplets, and enhance the film flow along GC corners.

Effect of operating conditions. - In this subsection, we examine the influences of several operating parameters plus the GC sidewall contact angle on liquid water flooding in the GC. Fig. 11 shows the effect of stoichiometric ratio on liquid water saturation distributions along the GC. It is seen that with the increase of stoichiometry, the droplet water saturation first reduces dramatically due to the enhanced gas drag force, and later on it reduces slowly due to the increased gas flow area at the GC cross section. This indicates that in practice the stoichiometry can be optimized to achieve a compromise between reducing droplet water saturation and decreasing gas pressure loss. The film water saturation for each stoichiometry is also plotted in Fig. 11. Interestingly we find no obvious difference among these film saturation distributions along the GC, which means that increasing stoichiometry has little influence on film flow along GC corners.

Fig. 12 displays the effect of operating current density on the water saturation and gas pressure distributions along the GC. The same

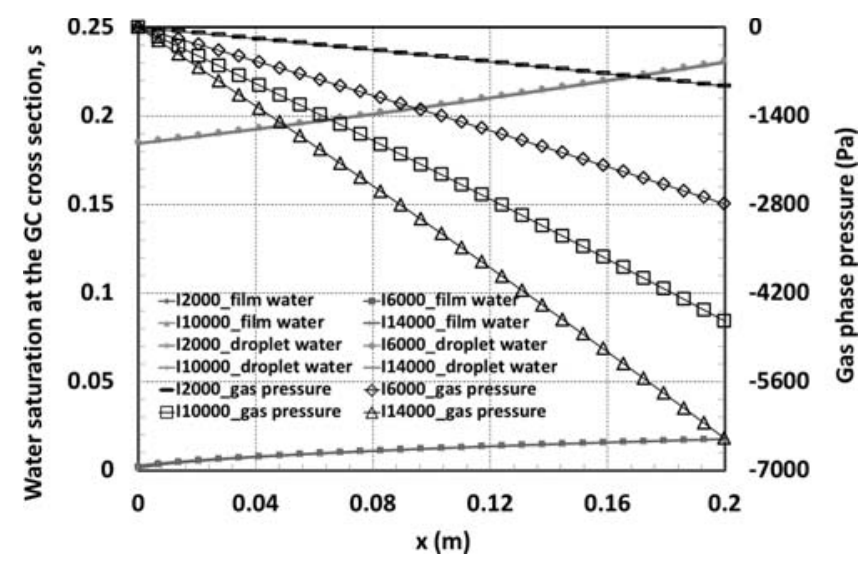

Figure 12. Distributions of water saturation and gas pressure along the GC for different operating current densities. 


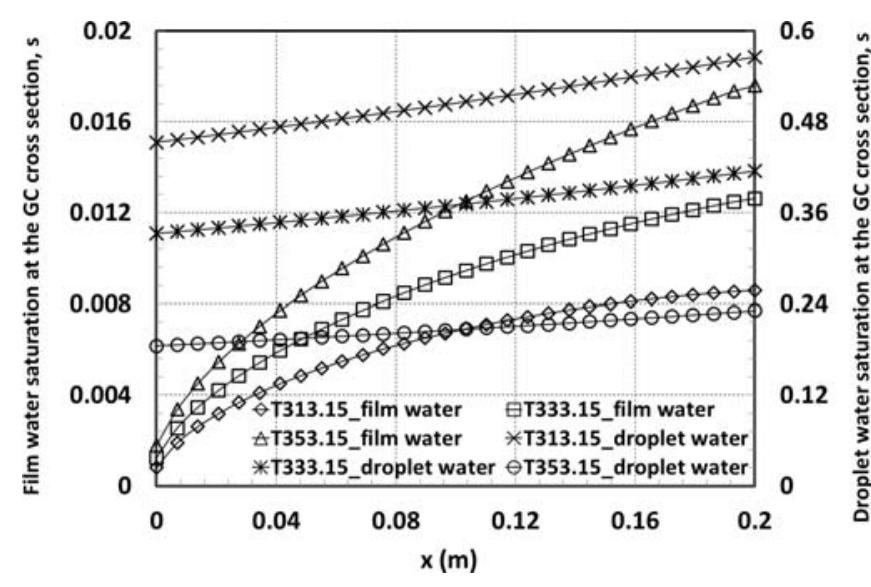

Figure 13. Distributions of water saturation (i.e. film water saturation and droplet water saturation) along the GC for different operating temperatures.

parameters as for the base case are employed except for varied current density and inclusion of droplet model. Interestingly, no discernible difference in either droplet water saturation or film water saturation distributions can be observed, although increasing current density means more liquid water is introduced into the GC. We can explain this phenomenon by the fact that the inlet gas flow rate increases as increasing the operating current density at a fixed stoichiometry of 2.0. Thus, the resultant gas drag force can always hold the GC water flooding at a certain level. The corresponding gas pressure loss along the GC as shown in Fig. 12 is directly proportional to the applied operating current density.

Fig. 13 displays the effect of operating temperature on the distributions of water saturation and gas pressure along the GC. As increasing the operating temperature, the oxygen molar density decreases for the fully humidified inlet air. In order to satisfy the fixed stoichiometric ratio of 2.0, the inlet gas flow rate increases with the increase of the operating temperature at the fixed current density of $6000 \mathrm{~A} / \mathrm{m}^{2}$. As a result, the droplet water saturation decreases as increasing the operating temperature as shown in Fig. 13. However, the film water saturation increases as increasing the operating temperature. This is attributed to the fact that water vapor condensation dominates the film water flooding along the GC corners (more and more water can be condensed from water vapor as increasing the operating temperature). Since droplet water flooding plays a dominant role in the gas pressure loss along the GC, the largest gas pressure loss of about $9300 \mathrm{~Pa}$ is found at the operating temperature of $313.15 \mathrm{~K}$, as shown in Fig. 14. Therefore, we conclude that liquid water flooding in GCs can

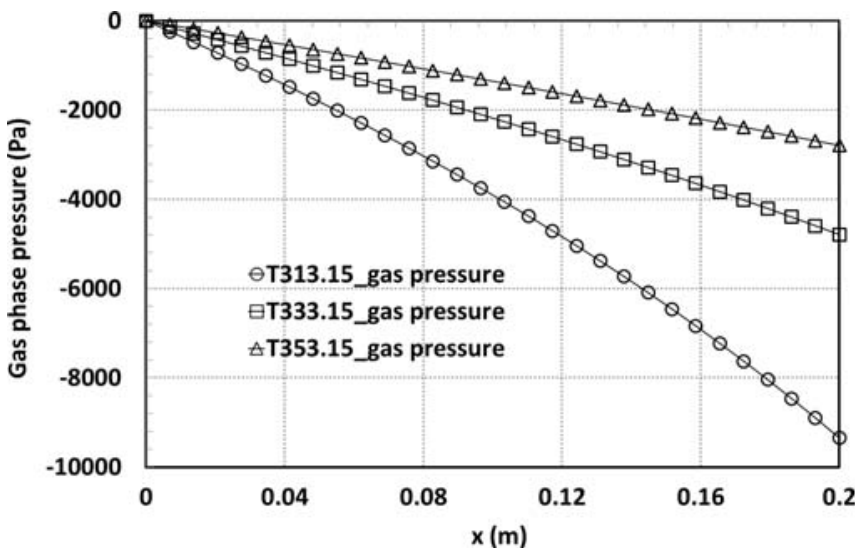

Figure 14. Effect of the operating temperature on the gas pressure drop along the GC.

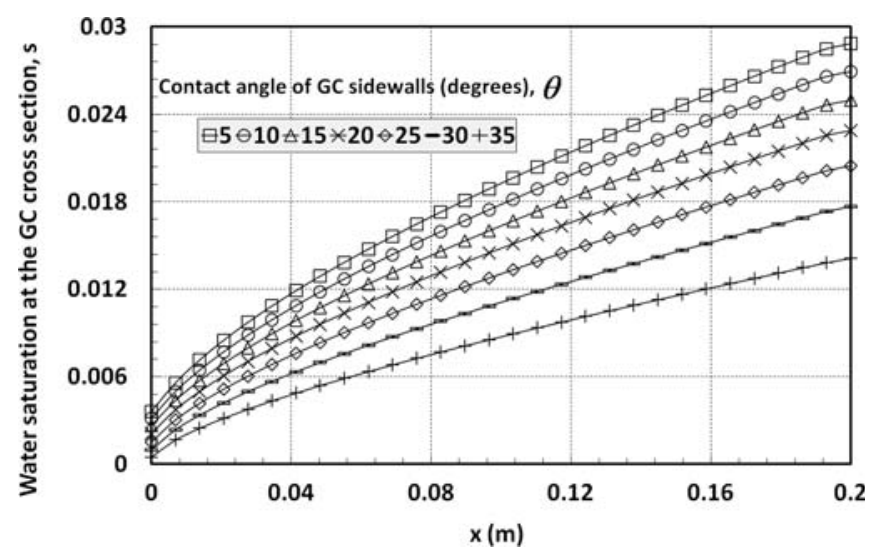

Figure 15. Effect of the sidewall contact angle of the GC on the film water distribution along the GC corners.

be considerably mitigated by increasing the cell operating temperature, which in addition gives rise to small gas pressure loss along GCs.

We also investigate the effect of the contact angle of the GC sidewalls on the water removal ability of the GC in the absence of droplet influence. Qin et al. ${ }^{29}$ studied the effect of the sidewall contact angle on the film flow at the GC corners by means of direct simulations. They concluded that larger contact angle of the hydrophilic sidewalls was beneficial to the film removal at the GC corners. However, less hydrophilic sidewalls were incapable of removing the water clogging in the GC efficiently. Based on our phenomenological model in this work, we also find that the water saturation at the GC cross section decreases with the increase of the contact angle of the GC sidewalls, as shown in Fig. 15. This is attributed to the fact that a larger contact angle of the hydrophilic sidewalls corresponds to smaller adhesion force on the film flow; in turn, less liquid water can be held in the GC. However, it should be pointed out that in practice a proper contact angle of the GC sidewalls should be selected to balance two requirements: $:^{37,38}$ increasing the film removal ability (less hydrophilicity), and removing the water clogging fast (higher hydrophilicity).

At last we note that the steady-state GC flooding model presented in this work is still at its early stage. Further improvements are still ongoing. For instance, a non-equilibrium phase change model needs to be introduced to consider the operation of partially humidified gas inlet. The model should be capable of modeling liquid water flooding in a large-scale flow field, probably including the effect of channel bends. Most importantly, extensive experimental data are needed to calibrate the droplet model proposed by the present work.

\section{Conclusions}

In this work, we first propose an idealized liquid water distribution at the GC cross section. Water droplets are assumed to form on the GDL surface, and water film resides at the GC corners. As a first attempt, we do not model the droplet dynamics explicitly, but we assume that droplets in the GC join the film flow along the GC corners through a series of dynamic processes. This assumption is quite reasonable under low gas flow rates. Then, we develop a phenomenological channel flooding model and a number of simulations are conducted to investigate the effects of droplets and several operating parameters on liquid water flooding in the GC. The following main conclusions are obtained:

1. The film water flow velocity in the GC is much smaller than that of the gas phase, which results in large gas drag force at the film-gas interface. This gas drag force assists in the film flow along GC corners considerably; thus playing a dominant role in the water removal along GC corners. In addition, the film flow along GC corners has little impact on gas pressure loss due to its small saturation. 
2. With the presence of droplets in the GC, the gas pressure loss is about three times as large as that without droplet effect at the stoichiometry of 2.0. As increasing the stoichiometry the gas pressure loss reduces first due to the enhanced gas drag force on the droplets. Later on the gas pressure loss increases slightly due to the enlarged gas flow rate, which begins to dominate the gas pressure loss along the GC. We find that droplets in the GC are the main contributions to the gas pressure loss and liquid water flooding. For better water management in GCs, we should depress the emergence of droplets, and enhance the film flow along GC corners.

3. In practice the stoichiometry can be optimized to obtain a compromise between reducing droplet water saturation and decreasing gas pressure loss. Particularly increasing stoichiometry has little influence on film flow along GC corners.

4. No discernible difference in either droplet water distribution or film water distribution can be found for different operating current densities. The gas pressure loss is directly proportional to the applied current density.

5. The liquid water in GCs can be considerably mitigated by increasing the cell operating temperature, which in addition gives rise to small pressure loss along GCs.

6. A proper contact angle of the GC sidewalls should be chosen to balance two requirements: increasing the film removal ability (less hydrophilicity), and removing the water clogging fast (higher hydrophilicity).

\section{Acknowledgments}

The first and second authors are members of the International Research Training Group NUPUS, financed by the German Research Foundation (DFG) and The Netherlands Organization for Scientific Research (NWO). We thank the anonymous reviewers for their contributions to improving the quality of the present work.

\section{Appendix A: Derivation of Film Flow Equation}

Gas drag force plays a dominant role ${ }^{29}$ in the film flow along GC corners, which must be included in the derivation of momentum equation. First, the point momentum equation for the film flow is given by Stokes equation

$$
\mu \nabla^{2} \vec{v}=\nabla p
$$

where $\mu$ is the dynamic viscosity of liquid water, $\vec{v}$ is the velocity vector at the microscopic scale, and $p$ is the pressure. We assume that the $y$ - and $z$-components of the water velocity and the second derivative of $\mathrm{x}$-component along the GC are zero. The pressure variation at the $\mathrm{GC}$ cross section is neglected. So, we get the film flow equation only in the channel direction

$$
\mu_{f}\left(\frac{\partial^{2} v_{x}}{\partial y^{2}}+\frac{\partial^{2} v_{x}}{\partial z^{2}}\right)=\frac{d p_{f}}{d x}
$$

Here, $\mu_{f}$ is the dynamic viscosity of liquid water, $v_{x}$ represents the film velocity along the $\mathrm{GC}$ (point velocity), and $p_{f}$ is the film pressure. We also can write down the corresponding boundary conditions as:

At the GC sidewalls:

$$
v_{x}=0
$$

At the gas-film interface

$$
\mu_{f} \frac{\partial v_{x}}{\partial \vec{n}}=\tau
$$

Eq. A3 shows the no slip boundary condition at the GC sidewalls. In Eq. A4, $\tau$ denotes the gas shear stress, and $\vec{n}$ is the unit vector normal to the gas-film interface pointing toward the gas phase. Then, we separate Eq. A2 into two new partial different equations (PDEs) associated with their boundary conditions ${ }^{31}$ :

$$
\left\{\begin{array}{l}
\mu_{f}\left(\frac{\partial^{2} v_{1}}{\partial y^{2}}+\frac{\partial^{2} v_{1}}{\partial z^{2}}\right)=\frac{d p_{f}}{d x} \\
\mu_{f}\left(\frac{\partial^{2} v_{2}}{\partial y^{2}}+\frac{\partial^{2} v_{2}}{\partial z^{2}}\right)=0
\end{array}\right.
$$

At the GC sidewalls:

$$
\left\{\begin{array}{l}
v_{1}=0 \\
v_{2}=0
\end{array}\right.
$$

At the gas-film interface:

$$
\left\{\begin{array}{l}
\frac{\partial v_{1}}{\partial \vec{n}}=0 \\
\mu_{f} \frac{\partial v_{2}}{\partial \vec{n}}=\tau \\
v_{x}=v_{1}+v_{2}
\end{array}\right.
$$

In Eq. A8, Film velocity along the GC is divided into two components: $v_{1}$ arising from the pressure gradient and $v_{2}$ due to the gas drag force.

To make Eq. A5 dimensionless, we define:

$$
\begin{gathered}
v_{1}=-\frac{r_{f}^{2}}{\mu_{f}} \frac{d p_{f}}{d x} v_{1}^{*} \\
y=r_{f} y^{*}, z=r_{f} z^{*} \\
v_{2}=\frac{r_{f} \tau}{\mu_{f}} v_{2}^{*}
\end{gathered}
$$

So, the dimensionless equations are obtained as:

$$
\left\{\begin{array}{l}
\frac{\partial^{2} v_{1}^{*}}{\partial\left(y^{*}\right)^{2}}+\frac{\partial^{2} v_{1}^{*}}{\partial\left(z^{*}\right)^{2}}=-1 \\
\mu_{f}\left(\frac{\partial^{2} v_{2}}{\partial\left(y^{*}\right)^{2}}+\frac{\partial^{2} v_{2}}{\partial\left(z^{*}\right)^{2}}\right)=0
\end{array}\right.
$$

At the GC sidewalls:

$$
\left\{\begin{array}{l}
v_{1}^{*}=0 \\
v_{2}^{*}=0
\end{array}\right.
$$

At the gas-film interface:

$$
\left\{\begin{array}{l}
\frac{\partial v_{1}^{*}}{\partial \vec{n}}=0 \\
\frac{\partial v_{2}^{*}}{\partial \vec{n}}=1
\end{array}\right.
$$

Based on the above derivations of the film flow at the microscopic scale, we integrate the point velocity $v_{x}$ over the film flow area at the GC cross section, and get:

$$
\begin{aligned}
v_{f} & =\frac{1}{A_{f}} \int_{A_{f}}\left(v_{1}+v_{2}\right) d s=\int_{A_{f}}\left(-\frac{r_{f}^{2}}{\mu_{f}} \frac{d p_{f}}{d x} v_{1}^{*}+\frac{r_{f} \tau}{\mu_{f}} v_{2}^{*}\right) d s \\
& =-\frac{r_{f}^{2}}{\mu_{f}} \frac{d p_{f}}{d x} \int_{A_{f}} v_{1}^{*} d s+\frac{r_{f} \tau}{\mu_{f}} \int_{A_{f}} v_{2}^{*} d s
\end{aligned}
$$

where $v_{f}$ is the average film flow velocity along the GC, and $A_{f}$ is the film flow area in the GC cross section. Next, we introduce two dimensionless parameters with the following definitions:

$$
\frac{1}{\beta}=\frac{1}{A_{f}} \int_{A_{f}} v_{1}^{*} d s, F_{d r a g}=\frac{1}{A_{f}} \int_{A_{f}} v_{2}^{*} d s
$$

in which, $\beta$ is called dimensionless viscous resistance, and $F_{\text {drag }}$ can be called dimensionless gas drag coefficient. Obviously, both dimensionless coefficients are related to the GC half corner angle and the contact angle of sidewalls. Finally we obtain the following film flow equation:

$$
v_{f}=-\frac{r_{f}^{2}}{\mu_{f} \beta} \frac{d p_{f}}{d x}+F_{d r a g} \frac{r_{f} \tau}{\mu_{f}}
$$

\section{List of Symbols}

a net water transfer coefficient

A area, $\mathrm{m}^{2}$

$b$ side edge length of GC cross section, $m$

C mass fraction 
$D$ molecular diffusivity, $\mathrm{m}^{2} \mathrm{~s}^{-1}$

$D_{H} \quad$ hydraulic diameter for gas flow, $\mathrm{m}$

$f_{t}$ effect of gas flow configuration on shear stress distribution

$F \quad$ Faraday's constant, $\mathrm{C} \mathrm{mol}^{-1}$

$F_{\text {drag }} \quad$ dimensionless gas drag coefficient

I current density, $\mathrm{A} \mathrm{m}^{-2}$

$L \quad$ interface length, $\mathrm{m}$

$M \quad$ molecular weight, $\mathrm{kg} \mathrm{mol}^{-1}$

$p$ pressure, $\mathrm{Pa}$

$P_{0} \quad$ cell operating pressure (i.e. gas inlet pressure), $\mathrm{Pa}$

$p_{\text {sat }} \quad$ saturated water vapor pressure, $\mathrm{Pa}$

$r_{f} \quad$ radius of curvature of gas-film interface, $\mathrm{m}$

$R \quad$ universal gas constant, $8.314 \mathrm{~J} \mathrm{~mol}^{-1} \mathrm{~K}^{-1}$

$R H \quad$ relative humidity

$S$ mass source term, $\mathrm{kg} \mathrm{m}^{-1} \mathrm{~s}^{-1}$

$\widehat{S} \quad$ molar source term, mol m $\mathrm{m}^{-1} \mathrm{~s}^{-1}$

$t$ ratio of GC down wall to reactive area

$T$ temperature, $\mathrm{K}$

$v \quad$ velocity, $\mathrm{m} \mathrm{s}^{-1}$

$w$ down edge length of GC cross section, $\mathrm{m}$

$x$ flow direction, $\mathrm{m}$

\section{Greek}

$\rho \quad$ mass density, $\mathrm{kg} \mathrm{m}^{-3}$

$\hat{\rho}$ molar density, mol m${ }^{-3}$

$\mu$ dynamic viscosity, $\mathrm{Pa} \mathrm{s}$

$\beta$ dimensionless viscous resistance

$\tau$ shear stress, $\mathrm{Pa}$

$\sigma \quad$ gas-liquid surface tension, $\mathrm{N} \mathrm{m}^{-1}$

$\alpha \quad$ upper half corner angle of GC, radian

$\theta$ contact angle of GC sidewalls, radian

$\theta_{d} \quad$ contact angle of GDL surface, radian

$r_{d} \quad$ droplet radius, radian

$\xi_{c} \quad$ cathodic stoichiometry

\section{Superscripts and Subscripts}

$\begin{aligned} g & \text { gas phase } \\ f & \text { film phase } \\ d & \text { droplet } \\ g c & \text { gas channel } \\ \mathrm{O}_{2} & \text { oxygen } \\ \mathrm{H}_{2} \mathrm{O} & \text { water vapor } \\ g w & \text { gas-wall } \\ g f & \text { gas-film } \\ g d & \text { gas-droplet } \\ \text { no-slip } & \text { wetted perimeter of gas flow area } \\ m & \text { membrane }\end{aligned}$

\section{References}

1. A. Z. Weber and J. Newman, Chem. Rev., 104, 4679 (2004).

2. N. Djilali, Energy, 32, 269 (2007).

3. S. S. Zhang, X. Z. Yuan, J. N. C. Hin, H. J. Wang, K. A. Friedrich, and M. Schulze, J. Power Sources, 194, 588 (2009).

4. M. M. Mench, Fuel Cell Engines, John Wiley \& Sons, Inc (2008).

5. C. Y. Wang, Chem. Rev., 104, 4727 (2004).

6. A. Bazylak, D. Sinton, and N. Djilali, J. Power Sources, 176, 240 (2008).

7. D. Spernjak, A. k. Prasad, and S. G. Advani, J. Powers Sources, 170, 334 (2007).

8. X. G. Yang, F. Y. Zhang, A. L. Lubawy, and C. Y. Wang, Electrochem. Solid-State Lett., 7, A408 (2004).

9. F. Y. Zhang, X. G. Yang, and C. Y. Wang, J. Electrochem. Soc., 153, A225 (2006).

10. P. K. Sinha, P. Halleck, and C. Y. Wang, Electrochem. Solid-State Lett., 9, A344 (2006).

11. C. Hartnig, I. Manke, R. Kuhn, N. Kardjilov, J. Banhart, and W. Lehnert, Appl. Phys. Lett., 92, 134106 (2008).

12. S. J. Lee, N. Y. Lim, S. Kim, G. G. Park, and C. S. Kim, J. Power Sources, 185, 867 (2008).

13. M. A. Hickner, N. P. Siegel, K. S. Chen, D. S. Hussey, D. L. Jacobson, and M. Arif, J. Electrochem. Soc., 155, B294 (2008).

14. M. A. Hickner, N. P. Siegel, K. S. Chen, D. S. Hussey, D. L. Jacobson, and M. Arif, J. Electrochem. Soc., 155, B427 (2008).

15. K. Yoshizawa, K. Ikezoe, Y. Tasaki, D. Kramer, E. H. Lehmann, and G. G. Scherer, J. Electrochem. Soc., 155, B223 (2008).

16. Y. Wang and K. S. Chen, J. Electrochem. Soc., 157, B1878 (2010).

17. K. Teranishi, S. Tsushima, and S. Hirai, J. Electrochem. Soc., 153, A664 (2006).

18. S. Lister, D. Sinton, and N. Djilali, J. Power Sources, 154, 95 (2006).

19. A. Bazylak, Liquid Water Transport in Fuel Cell Gas Diffusion Layers, Ph.D. Thesis, University of Victoria, 2008.

20. C. Hartnig, I. Manke, R. Kuhn, S. Kleinau, J. Goebbels, and J. Banhart, J. Power Sources, 188, 648 (2009).

21. Z. Lu, S. G. Kandlikar, C. Rath, M. Grimm, W. Domigan, A. D. White, M. Hardbarger, J. P. Owejan, and T. A. Trabold, Int. J. Hydrogen Energy, 34, 3445 (2009).

22. Y. H. Cai, J. Hu, H. P. Ma, B. L. Yi, and H. M. Zhang, J. Power Sources, 161, 843 (2006).

23. X. Zhu, P. C. Sui, and N. Djilali, J. Power Sources, 181, 101 (2008).

24. J. Y. Chio and G. H. Son, J. Mech. Sci. Technol., 22, 2590 (2008).

25. G. F. Pinder and W. G. Gray, Essentials of Multiphase Flow and Transport in Porous Media, John Wiley \& Sons, 2008.

26. T. Berning, M. Odgaard, and S. K. Kær, J. Electrochem. Soc., 156, B1301 (2009).

27. S. Basu, J. Li, and C. Y. Wang, J. Power Sources, 187, 431 (2009).

28. C. Z. Qin, D. Rensink, S. Fell, and S. M. Hassanizadeh, J. Power Sources, 197, 136 (2012).

29. C. Z. Qin, D. Rensink, S. M. Hassanizadeh, and S. Fell, J. Electrochem. Soc., 159(4), B434 (2012).

30. V. Joekar-Niasar, the Immiscibles: Capillary Effect in Pours Media - Pore-network Modeling, Ph.D. Thesis, Utrecht University, 2011.

31. D. A. Caulk, J. Fuel Cell Sci. Technol., 7, 031013 (2010).

32. T. C. Ransohoff and C. J. Radke, J. Colloid Interface Sci., 121, 392 (1988)

33. P. K. Sinha and C. Y. Wang, Electrochim. Acta 52, 7936 (2007).

34. Q. Ye and T. V. Nguyen, J. Electrochem. Soc., 154, B1242 (2007)

35. D. Zhou, M. Blunt, and F. M. Orr, J. Colloid Interface Sci., 187, 11 (1997).

36. E. Kimball, T. Whitaker, Y. G. Kevrekidis, and J. B. Benziger, AIChE J., 54, 1313 (2008).

37. H. Y. Tang, A. Santamaria, J. W. Park, C. Lee, and W. Hwang, J. Power Sources, 196, 9373 (2011).

38. A. Turhan, S. Kim, M. Hatzell, and M. M. Mench, Electrochim. Acta, 55, 2734 (2010).

39. I. S. Hussaini and C. Y. Wang, J. Power Sources, 187, 444 (2009). 